Universidade Tecnológica Federal do Paraná - UTFPR

Campus Ponta Grossa - Paraná - Brasil

ISSN: 1981-3686 / v. 03, n. 02: p. 75-80, 2009
Revista Brasileira deTecnologia

Agroindustrial

\title{
ESTUDO DO COMPORTAMENTO DO HIDROXIMETILFURFURAL (HMF) EM MÉIS ARMAZENADOS EM RECIPIENTES DE PLÁSTICO E METAL
}

\section{STUDY OF THE BEHAVIOR OF HYDROXYMETHYLFURFURAL (HMF) IN HONEY STORED IN PLASTIC CONTAINERS AND METAL}

Kelly de Fátima Nogueira Lima Silva ${ }^{1}$; Dyego da Costa Santos ${ }^{1}$; Charles Teles Santos da Silva ${ }^{2}$; Alexandre José de Melo Queiroz ${ }^{2 ;}$ Afonso Odério Nogueira Limae ${ }^{3}$

${ }^{1}$ Faculdade de Tecnologia - CENTEC - Limoeiro do Norte - Brasil fafa_charles13@yahoo.com.br

${ }^{2}$ Universidade Federal de Campina Grande - UFCG - Brasil charles_fafa@yahoo.com.br

${ }^{3}$ Universidade Federal do Ceará - UFC - Brasil oderio@bisanet.com.br

\section{Resumo}

O presente trabalho foi conduzido no Laboratório de Bromatologia da FATEC de Limoeiro do Norte-CE, durante os meses de junho a dezembro de 2004. O objetivo foi avaliar o comportamento do hidroximetilfurfural (HMF) do mel de Apis mellifera durante o armazenamento de seis meses em recipientes de plástico e de metal. Os méis utilizados neste trabalho foram obtidos no mês de junho de 2004, a partir de coletas realizadas por apicultores no município de Limoeiro do Norte-CE $e$ acondicionados em dois tipos de embalagens a granel. O armazenamento ocorreu a temperatura ambiente (média de $30^{\circ} \mathrm{C}$ ), no Apiário Altamira, situado no distrito de Bixopá, município de Limoeiro do Norte - CE. Mensalmente foram realizadas coletas das amostras de méis dos dois tipos de embalagens para realização das análises de hidroximetilfurfural, que foi determinado de acordo com Lanara (1981). O valor médio de HMF obtido no experimento foi de 31,29 mg/kg para uma variação de 5,28 a 72,21 mg/kg. O teor de HMF nas amostras armazenadas foi influenciado pelo tempo, aumentando nas duas embalagens. O tempo de armazenamento influenciou o HMF com diferente intensidade nos períodos de 60, 90, 120,150 e 180 dias, conforme o tipo de embalagem, com os níveis na embalagem lata sempre superior. De acordo com os resultados podese concluir que, dentre as duas embalagens utilizadas no estudo, o recipiente plástico apresentou melhor resultado na manutenção dos níveis de HMF.

Palavras-chave: mel, hidroximetilfurfural, armazenamento.

\section{Introdução}

Cuidados especiais devem ser tomados em relação ao armazenamento, tanto do mel a granel (baldes plásticos e tambores) como do fracionado (embalagens para o consumo final), em relação à higiene do ambiente e, principalmente, em relação ao controle da temperatura. Altas temperaturas durante todo o processamento e estocagem são prejudiciais à qualidade do produto final, uma vez que o efeito nocivo causado ao mel é acumulativo e irreversível. Essas embalagens devem ser colocadas sobre estrados de madeira ou outro material, impedindo o contato direto com o piso e facilitando seu deslocamento no caso da utilização de empilhadeiras (Pereira, 2003). 
Em embalagens a granel (25 kg), os baldes plásticos têm relação custo-benefício superior ao da lata de metal, além de proporcionarem facilidade no transporte (presença de alças). Já para capacidades superiores (300 kg), destinadas à exportação, a embalagem usada é o tambor de metal (com revestimento interno de verniz especial). Quanto às embalagens para o varejo, tanto o plástico, específico para alimentos, como o vidro são recomendáveis, embora o vidro seja o material ideal para o acondicionamento do mel, inclusive como único material aceito para a exportação (mel fracionado) e para a certificação orgânica (Pereira, 2003).

O objetivo do trabalho foi avaliar o comportamento do hidroximetilfurfural (HMF) do mel coletado no município de Limoeiro do Norte durante o armazenamento (180 dias) em recipientes de plástico e de metal.

\section{Metodologia}

\subsection{Obtenção do mel}

Os méis utilizados neste trabalho, produzidos por Apis mellífera L. com predominância da florada de vassourinha de botão, foram obtidos no mês de junho de 2004, a partir de coletas realizadas por apicultores no município de Limoeiro do Norte, localizado na Região do Baixo Jaguaribe - CE.

As melgueiras, ao chegarem à casa de mel, foram colocadas sobre bandejas de aço inoxidável e levadas para a sala de recepção dos favos; em seguida foram depositadas em área isolada do recinto, onde ocorreu a extração do mel e as outras etapas do processamento. Essas melgueiras provenientes do campo não tiveram acesso à área de manipulação; assim, apenas os quadros foram transportados para a sala de processamento.

As etapas de desoperculação dos quadros, centrifugação, filtragem e decantação do mel foram executadas de acordo com as normas higiênico-sanitárias indicadas pelas Boas Práticas de Fabricação (BPF). Após a desoperculação dos favos, os quadros foram encaminhados para a centrífuga, onde se iniciou lentamente o processo, aumentando-se a sua velocidade progressivamente para não quebrar os quadros que estão cheios de mel. Uma vez extraído, o mel foi retirado da centrífuga por um sistema de bombeamento, escoando-o diretamente para o decantador iniciando-se assim o processo de filtragem. Após a filtragem, o mel foi encaminhado para o decantador, onde "descansou" por 48 horas, a fim de que as eventuais partículas que não foram retiradas pela filtragem e as bolhas criadas durante o processo se desloquem para a porção superior do decantador, sendo retiradas posteriormente durante o procedimento de envase. 
Após o processamento, o mel foi acondicionado em dois tipos de embalagens a granel, recipiente plástico, do tipo balde e recipiente metálico, do tipo lata, com 25kg cada. Em seguida, as amostras assim acondicionadas foram colocadas sobre estrados de madeira e armazenadas durante seis meses à temperatura ambiente (média de $30^{\circ} \mathrm{C}$ ), no Apiário Altamira, situado no distrito de Bixopá, município de Limoeiro do Norte - CE.

Mensalmente foram realizadas coletas das amostras de méis dos dois tipos de embalagens para realização das análises de hidroximetilfurfural. Para coletar as amostras para as análises as embalagens eram abertas rapidamente, no máximo por 1 minuto, e retiradas alíquotas, com uma concha de aço inoxidável, em três profundidades: superfície, meio e fundo das embalagens. As amostras assim coletadas eram colocadas em recipientes de vidro codificados, com capacidade para $700 \mathrm{~g}$, de acordo com tipo de embalagem.

\subsection{Método de Análise}

As determinações analíticas foram conduzidas no Laboratório de Bromatologia da Faculdade de Tecnologia CENTEC de Limoeiro do Norte-CE durante os meses de junho a dezembro de 2004.

O teor de HMF dos méis armazenados em embalagens plástica e metálica por 180 dias foi determinado quantitativamente, na qual o princípio da análise se baseia que em meio ácido o ácido barbitúrico condensa-se com o HMF formando um composto de coloração vermelha, de acordo com Lanara (1981).

As amostras foram enquadradas na Instrução Normativa nº11 (Brasil, 2000), que permite um valor máximo de HMF de 60mg/kg de mel.

\section{Resultados e Discussão}

A Tabela 1 exibe os resultados da análise de variância para o percentual de HMF, onde se observa, através do teste $\mathrm{F}$, efeito significativo ao nível de $1 \%$ de probabilidade para os fatores embalagem, tempo e para a interação entre os dois fatores.

Tabela 1 - Análise de variância do hidroximetilfurfural (mg HMF/kg) para méis de florada de vassourinha de botão, nos diferentes tipos de embalagens e submetido a seis meses de armazenamento. Apiário Altamira, Bixopá Limoeiro do Norte, CE. 


\begin{tabular}{lcccc}
\cline { 2 - 4 } & G.L & S.Q & Q.M & F \\
\hline Embalagem & 1 & 53,37991 & 53,37991 & $90,6656 * *$ \\
Tempo & 6 & 41016,80469 & 6836,13428 & $11611,1582 * *$ \\
Emb. x Tempo & 6 & 28,05603 & 4,67600 & $7,9422 * *$ \\
Resíduo & 56 & 32,97031 & 0,58876 & \\
\hline
\end{tabular}

Total

69

41131,21094

** - significativo ao nível de $1 \%$ de probabilidade

O valor médio de hidroximetilfurfural Tabela 2 obtido no experimento foi de 31,29 mg/kg para uma variação de 5,28 a 72,21 mg/kg. O teor de HMF nas amostras armazenadas foi influenciado pelo tempo, aumentando nas duas embalagens. O tempo de armazenamento influenciou o HMF com diferente intensidade nos períodos de 60, 90, 120,150 e 180 dias, conforme o tipo de embalagem, com os níveis na embalagem lata sempre superior. A norma vigente (Brasil, 2000) estabelece um máximo de $60 \mathrm{mg} / \mathrm{kg}$ para o hidroximetilfurfural. Sendo assim, os méis nas duas embalagens, aos 180 dias de armazenamento, estavam fora do padrão de qualidade com relação a esse parâmetro.

Tabela 2 - Valores médios de hidroximetilfurfural (mg HMF/kg) para méis de florada de vassourinha de botão. Interação embalagem x tempo de armazenamento.

\begin{tabular}{cccccccc}
\hline \multirow{2}{*}{ Embalagem } & \multicolumn{7}{c}{ Tempo (dia) } \\
\cline { 2 - 7 } & 0 & 30 & 60 & 90 & 120 & 150 & 180 \\
\hline Balde & $5,40 \mathrm{aG}$ & $8,02 \mathrm{aF}$ & $10,37 \mathrm{bE}$ & $21,66 \mathrm{bD}$ & $41,70 \mathrm{bC}$ & $57,38 \mathrm{bB}$ & $68,35 \mathrm{bA}$ \\
Lata & $5,28 \mathrm{aG}$ & $8,55 \mathrm{aF}$ & $11,44 \mathrm{aE}$ & $23,61 \mathrm{aD}$ & $43,80 \mathrm{aC}$ & $60,23 \mathrm{aB}$ & $72,21 \mathrm{aA}$ \\
\hline
\end{tabular}

DMS p/ colunas $=0,97 ;$ DMS p/ linhas $=1,48 ; \mathrm{MG}=31,28 \mathrm{mg} / \mathrm{kg} ; \mathrm{CV}=2,45 \%$.

DMS: Desvio mínimo significativo; MG: Média geral e CV: Coeficiente de variação.

Obs.: médias seguidas pela mesma letra minúscula nas colunas e maiúsculas nas linhas não diferem estatisticamente pelo teste de Tukey, a 5\% de probabilidade.

Os resultados obtidos no presente trabalho foram superiores aos obtidos por Almeida (2002), que, estudando méis poliflorais do cerrado paulista, encontrou uma variação de 0,0 a $11,45 \mathrm{mg}$ $\mathrm{HMF} / \mathrm{kg}$ e com um valor médio de 3,70 mg HMF/kg.

Na Figura 1 têm-se os valores médios do HMF do mel em função do tempo de armazenamento. Observa-se o aumento nos resultados de HMF com o fator tempo, atingindo-se valores progressivamente mais altos com o decorrer do armazenamento, com os valores experimentais das amostras acondicionadas em lata superando, os das amostras em balde. 


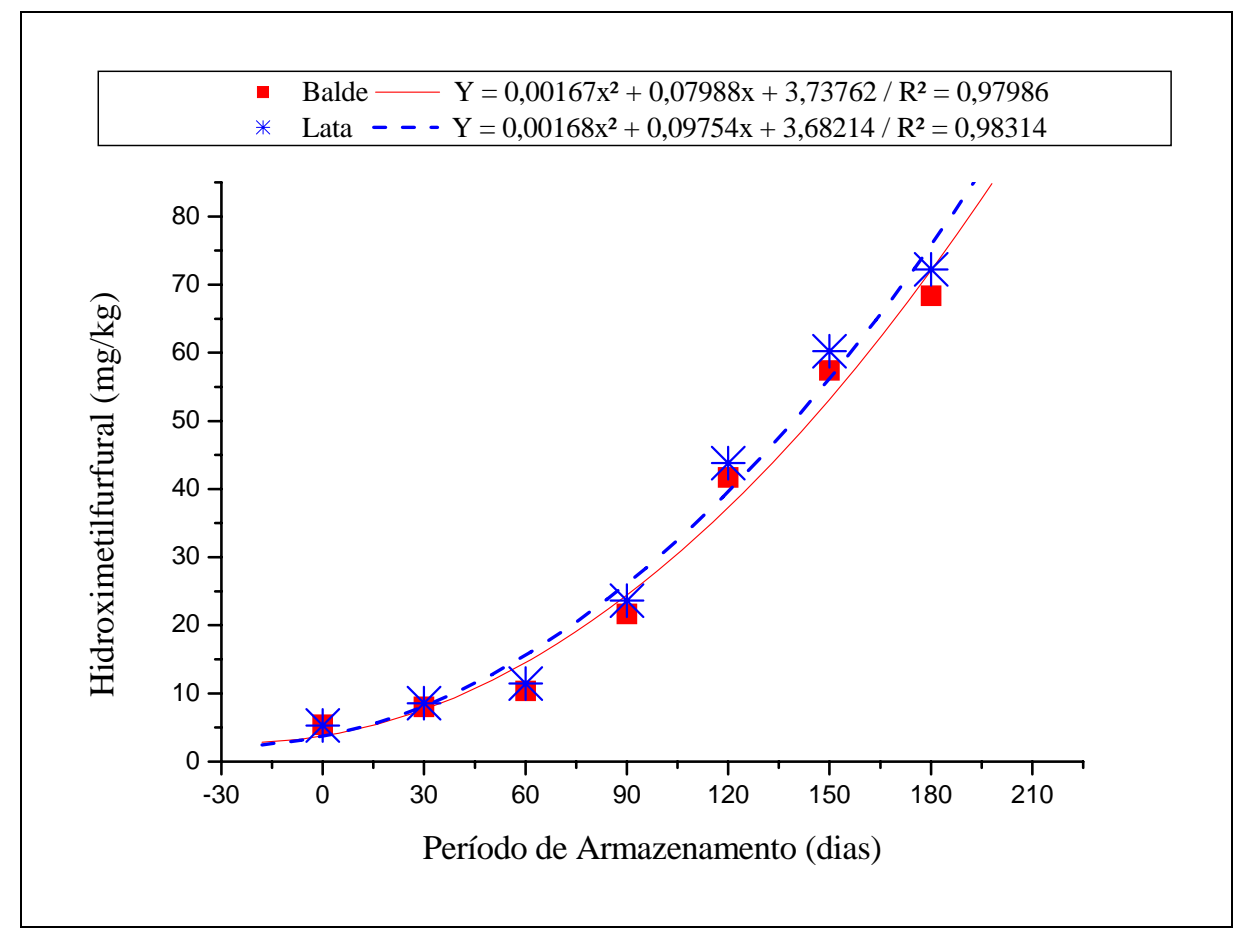

Figura 1 - Hidroximetilfurfural para o mel produzido a partir da florada de vassourinha de botão durante o armazenamento nas diferentes embalagens.

\title{
4. Conclusão
}

No quinto mês (150 dias) de armazenamento a embalagem de metal apresentou valor de hidroximetilfurfural superior a 60mg/kg (Brasil, 2000) e a embalagem plástica apresentou valor de HMF superior ao permitido pela legislação no sexto (180 dias) mês de armazenamento. Dentre as duas embalagens utilizadas no estudo, o recipiente plástico apresentou melhor resultado na manutenção dos níveis de hidroximetilfurfural.

\begin{abstract}
This study was conducted at the Laboratory of Food Science, Faculty of Technology CENTEC Limoeiro do Norte-CE during the months of June to December 2004. The objective was to evaluate the behavior of hydroxymethylfurfural (HMF) in honey collected in the municipality of Limoeiro do Norte-CE during the six months storage in plastic and metal. The honey used in this work produced by Apis mellifera L., were obtained in June 2004 from collections made by beekeepers in the municipality of Limoeiro do Norte-CE. The same were placed in two types of bulk packaging, container type plastic bucket and a metal container, the type can, with $25 \mathrm{~kg}$ each. Then the samples were placed so packed on wooden pallets and stored for six months at room temperature (average $30^{\circ} \mathrm{C}$ ), the Bee Altamira, located in the district Bixopá, city of Limoeiro do Norte-CE. Monthly collections were made of honey samples of two types of packaging to carry out the analysis of hydroxymethylfurfural. Samples thus collected were placed in glass containers coded with a capacity of $700 \mathrm{~g}$, according to type of packaging. The hydroxymethylfurfural content of honeys was determined quantitatively, in which the principle of analysis that is based on an acid barbituric acid condenses with the HMF forming a composite of red color, according to Lanara (1981). The average value of hydroxymethylfurfural obtained in the experiment was $31.29 \mathrm{mg} / \mathrm{kg}$ to a range
\end{abstract}


from 5.28 to $72.21 \mathrm{mg} / \mathrm{kg}$. The content of HMF in the samples was influenced by storage time, increasing in the two packages. The storage time influenced the HMF with different levels of intensity during periods of 60, 90, 120.150 and 180 days, depending on the type of packaging, with levels in the package can always superior. According to the results we can conclude that among the two packages used in the study, the plastic container showed better results in maintaining the levels of HMF.

Keywords: honey, hydroxymethylfurfural (HMF), storage

\section{Referencias Bibliográficas}

Almeida, D. de. Espécies de abelhas (Hymenoptera, Apoidea) e tipificação dos méis por elas produzidos em área de cerrado do município de Pirassununga, Estado de São Paulo. 2002. 103 f. Dissertação (Mestrado em Ciências) Escola Superior de Agricultura “Luiz de Queiroz”, Universidade de São Paulo, Piracicaba.

Brasil. Ministério da Agricultura. Instrução normativa $\mathbf{n}^{\mathbf{0}} \mathbf{1 1}$, de 20 de outubro de 2000 . Estabelece o regulamento técnico de identidade e qualidade do mel. Diário Oficial da República Federativa do Brasil, Poder Executivo, Brasília, DF, 23 out. 2000. Seção 1, p. 16-17.

LANARA - Laboratório Nacional de Referencia Animal. Métodos analíticos oficiais para controle de produtos de origem animal e seus ingredientes: II - métodos físicos e químicos. Brasília: Ministério da Agricultura, 1981.

Pereira, F. de M.; Lopes, M. T. do R.; Camargo, R. C. R. de.; Vilela, S. L. de O. Produção de mel. Sistema de produção 3 (Versão eletrônica). Embrapa Meio-Norte, 2003.

Nome: Kelly de Fátima Nogueira Lima Silva

Filiação institucional: Faculdade de Tecnologia CENTEC - Limoeiro do Norte

Departamento: Tecnologia de Alimentos

Função ou cargo ocupado: Professora

Titulação: Mestre em Engenharia Agrícola - UFCG

Endereço completo para correspondência:

Avenida Dom Aureliano Matos, 1007, Apto. 102. Centro, Limoeiro do Norte-CE, Brasil. CEP: 62930-000

Telefones para contato: (88) 99217613

e-mail: fafa_charles13@yahoo.com.br 